\title{
Glossary of Neurostimulation Terminology: A Collaborative Neuromodulation Foundation, Institute of Neuromodulation, and International Neuromodulation Society Project
}

\author{
Richard B. North, $\mathrm{MD}^{1}$; Scott F. Lempka, $\mathrm{PhD}^{2}$; Yun Guan, MD, $\mathrm{PhD}^{3,4}$; \\ Ellen L. Air, MD, $\mathrm{PhD}^{5}$; Lawrence R. Poree, $\mathrm{MD}^{2} \mathrm{PhD}^{6}$; Jane Shipley, $\mathrm{BA}^{1}$; \\ Jeffrey Arle, $\mathrm{MD}, \mathrm{PhD}^{7}$; Philippe Rigoard, $\mathrm{MD} \mathrm{PhD}^{8}$; Simon Thomson, $\mathrm{MBBS}^{9}$
}

\begin{abstract}
Objective: Consistent terminology is necessary to facilitate communication, but limited efforts have addressed this need in the neurostimulation community. We set out to provide a useful and updated glossary for our colleagues and prospective patients.

Materials and Methods: This collaborative effort of the Neuromodulation Foundation (NF), the Institute of Neuromodulation (IoN), and the International Neuromodulation Society (INS) expands a glossary first published in 2007 for spinal cord stimulation. Peripheral nerve, dorsal root ganglion, deep brain, and motor cortex stimulation have been added to our scope. Volunteers from the collaborating entities used a nominal group process, consensus development panels, and the Delphi technique to reach consensus on inclusion and definition of terms. We created a glossary suitable for print and for expansion on the websites of the collaborating entities, which will offer the possibility of explaining definitions for a general audience. We excluded proprietary and brand names but included terms that have attracted proprietary interest without becoming brands or trademarks. We made an effort to be inclusive while also being concise and economical with space.

Results: We identified and defined 91 terms for this print edition and created an accompanying list of acronyms. As appropriate, we provided figures to illustrate the definitions.

Conclusions: Although we refer to the glossary presented herein as the print edition, it can of course be viewed and searched electronically. NF, loN, and INS will continue to collaborate on expanded web editions that can include hyperlinks for internal and external navigation. We believe this glossary will benefit our growing field by facilitating communication and mitigating inappropriate use of neurostimulation terms.
\end{abstract}

Keywords: Glossary, neuromodulation, neurostimulation, taxonomy, terminology

Conflict of Interest: Richard B. North serves as the unpaid President of the nonprofit Institute of Neuromodulation (supported by the North American Neuromodulation Society) and of the nonprofit Neuromodulation Foundation, Inc, to which Abbott, Boston Scientific, Medtronic, Nevro, Nuvectra, and Stimwave have provided grants and support. His former employers, the Johns Hopkins University (until 2007) and Sinai Hospital (until 2013), also received support from the companies active in SCS at the time. Stimwave has paid him for expert testimony, and he has received support for his attendance from meeting organizers (not thirdparty sponsors) in addition to royalties from Abbott and consulting fees and royalties from Nuvectra. His wife holds shares in Stimwave. Scott F. Lempka reports grant support from Medtronic and Presidio Medical. He has served as a scientific advisory

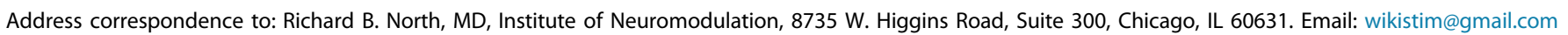

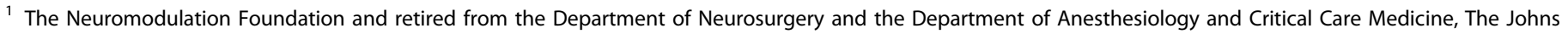
Hopkins University School of Medicine, Baltimore, MD, USA;

2 Department of Biomedical Engineering, University of Michigan, Ann Arbor, MI, USA;

3 Department of Anesthesiology and Critical Care Medicine, The Johns Hopkins University School of Medicine, Baltimore, MD, USA;

4 Department of Neurological Surgery, The Johns Hopkins University School of Medicine, Baltimore, MD, USA;

5 Department of Neurosurgery, Henry Ford Health System, Detroit, MI, USA;

6 Division of Pain Medicine, Department of Anesthesia and Perioperative Care, University of California, San Francisco, San Francisco, CA, USA;

7 Department of Neurosurgery, Harvard Medical School, Boston, MA, USA;

8 Department of Spine Surgery \& Neuromodulation, Poitiers University Hospital, Poitiers, France; and

9 Department of Pain Management and Neuromodulation, Mid and South Essex University Hospitals NHSFT, Essex, UK

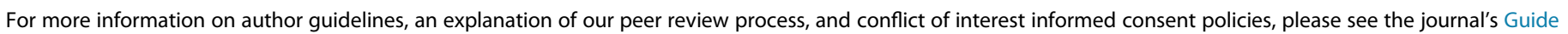
for Authors.

Source(s) of financial support: The meetings were virtual and hosted by the Neuromodulation Foundation and the Institute of Neuromodulation. 
board member for Abbott and Presidio Medical and holds stock or stock options in Hologram Consultants and Presidio Medical. Yun Guan reports no conflict of interest. Ellen L. Air reports grant support from Medtronic and consulting fees from Stryker and from Functional Neuromodulation, for which she has participated in a clinical event committee. She holds leadership roles in the American Society for Stereotactic and Functional Neurosurgery and the Congress of Neurological Surgeons. Lawrence R. Poree has received consulting fees from Medtronic, Nalu, and Saluda. He holds stock or stock options in Saluda and Nalu. Jane Shipley is an employee of the nonprofit Neuromodulation Foundation, Inc (since 2007), to which Abbott, Boston Scientific, Medtronic (Abbott), Nevro, Nuvectra, and Stimwave have provided grants and support. Her former employer (until 2007), the Johns Hopkins University, also received support from the companies active in SCS at the time. Any support she was offered for participation in meetings has instead gone directly to the Neuromodulation Foundation. Jeffrey Arle reports no conflict of interest. Philippe Rigoard reports grants and consulting fees from Medtronic, Abbott, and Boston Scientific and consulting fees from Grunhental, Spineart, and Allergan. He has received compensation for participating in educational events from ECMT (Medronic) and Boston Scientific. Simon Thomson has received consulting fees and payment or honoraria for participating in education events from Boston Scientific. He has also been on a data safety monitoring or advisory board for Galvani Bioelectronics.

\section{INTRODUCTION}

"In the first place, there must be a group of physical symbols, such as words . . . which by general agreement convey certain meanings to the parties communicating," wrote Hartley in 1928 in one of the foundational documents of information theory. ${ }^{1}$

Neuromodulation is a multidisciplinary field involving neurobiology, medicine, and engineering, inter alia, and as such, it uses terminology and vocabulary from multiple sources. Ongoing advances, for example, the development of novel stimulation waveforms, continue to add complexity. Publications in the field appear in the professional journals and textbooks of multiple specialties as well as on websites accessible to the general public. This diverse community needs a unified language to facilitate communication, avoiding ambiguities and misunderstanding.

To date, limited efforts have addressed this need for the field of neuromodulation, for example, a spinal cord stimulation (SCS) glossary published in print in 2007 and kept up to date on a website $^{2,3}$ and a "glossary-of-definitions" section on another website that emphasizes the conditions treated, as opposed to technical terminology. ${ }^{4}$ Neuroscience textbook glossaries are relevant and helpful but do not include our specialized engineering and clinical terminology. ${ }^{5}$ Therefore, as described in this article, we have undertaken to provide a useful resource for our colleagues and prospective patients, specific to our field and to their needs. We hope to facilitate communication not only between authors and peer reviewers contributing to the neuromodulation literature, including physicians, scientists, and engineers, but also among health care providers, payers, industry, and the general public.

\section{MATERIALS AND METHODS}

Our starting point was the latest version of the Glossary from the "Practice Parameters for the Use of Spinal Cord Stimulation in the Treatment of Chronic Neuropathic Pain," which was first published (in print) as a supplemental issue in Pain Medicine in $2007^{2}$ and has since been maintained on the Neuromodulation Foundation (NF) website. $^{3}$ Ongoing efforts to update this practice guide include expanding its scope to include dorsal root ganglion (DRG) and peripheral nerve stimulation (PNS) along with SCS. In parallel with this, the vision statement of the Institute of Neuromodulation (IoN), which was established in 2017, seeks to "Improve the understanding" of the field. ${ }^{6}$ To this end, in 2019 to 2020, the loN began a glossary/taxonomy project in collaboration with the NF, expanding the scope beyond SCS, DRG, and PNS to include vagus nerve, deep brain, and motor cortex stimulation and introducing international authorship. In 2021, the International Neuromodulation Society (INS) ${ }^{4}$ joined the program, and we undertook to reconcile and improve the glossaries.

The resulting glossary was developed by a collaboration among leaders and members of the NF, loN, and INS in a series of meetings conducted online and complemented by email correspondence between January and July 2021 (during the COVID-19 pandemic). As a consensus process, development followed three common methods in aggregate: nominal group process, consensus development panels, and use of the Delphi technique. ${ }^{7,8}$ The average meeting size slightly exceeded the number of authors, who were determined by standard International Committee of Medical Journal Editors criteria. ${ }^{9}$

The focus of this glossary is terminology specific to our field, including terms that have been imprecisely or inappropriately used in print and those whose definition is clouded by ambiguity or confusion. This print edition of the glossary is subject to space constraints and is static; accordingly, to accommodate the need for ongoing revisions and maintenance as well as to expand definitions for the general public, we determined and agreed at the outset that we would publish an online version or versions on the websites of the NF, loN, and/or the INS. Web editions, therefore, will be a superset of this print edition and will include entries collected in parallel but excluded because of print size constraints. Furthermore, we determined that we will link the online glossary to existing resources, for example, glossaries in neuroscience textbooks, ${ }^{5}$ as appropriate to avoid unnecessary duplication.

This print edition of the glossary has been prepared for the readership of Neuromodulation, but among our potential online users are the lay public, for example, prospective patients who have been presented with commercial and/or medical literature, and the readership of accessible neuromodulation websites (for example, the $\mathrm{NF}^{3}$ and INS $^{4}$ websites). These readers might be unfamiliar with neuromodulation, and their educational backgrounds will vary. Accordingly, to the extent possible, we use language appropriate for a broad audience. We sought reviewers with neither advanced degrees nor special expertise to assist with readability. Their suggestions will be most valuable in the online editions that have space for lengthy explanations.

Proprietary and brand names customarily are mentioned once and only once in the Methods section of medical and scientific publications. They were not included in this glossary, of course, but the glossary should be useful in describing the design features 
Table 1. Neurostimulation Glossary.

Accommodation - a rise in neuron membrane threshold with depolarization or a fall with hyperpolarization. The rate and degree are related to axon diameter and play a role in underlying mechanisms of SCS. (A secondary definition is adaptation, habituation, or reduced attention to a steady, unchanging stimulus, such as declining pain relief from SCS; see Tolerance.)

Action potential - a rapid rise and fall in voltage gradients, caused when resting potential across a neuronal membrane is depolarized sufficiently. An action potential can propagate over distance (most rapidly along a myelinated axon) and can be triggered or inhibited by electrical stimulation.

Active electrode (or contact) - electrode contact(s) at or near a targeted structure, through which current passes to achieve the physiologic effect(s) of stimulation.

Afferent - nerve fiber(s) conducting or carrying signals to a structure (for example, a nucleus) in the spinal cord or brain (for example, from peripheral tissues or organs).

Amplitude - voltage or current, typically the peak value in a waveform.

Anisotropic - having different properties or values in different directions (that is, the opposite of isotropic). The spinal cord and peripheral nerves, for example, are anisotropic in that they have greater impedance measured transversely than they do longitudinally.

Anode - a stimulating contact programmed as a positive pole that attracts anions (negative ions) and through which positive current enters the body.

Antidromic - a signal or molecule traveling in the opposite direction to that which is normal in a nerve fiber or transduction pathway; the opposite of orthodromic.

Array (as in electrode array) - an assembly of multiple stimulating/recording elements and contacts. A percutaneous electrode, for example, is configured as a longitudinal array (single column) of contacts. Parallel longitudinal arrays can be created with parallel percutaneous electrodes, or a plate/paddle electrode can be prefabricated with contacts in two or more columns with fixed separation and offsets (Fig. 1).

Battery - a power cell or device used to store and provide electrical energy by electrochemical means. In the vernacular, although it is just one component of an implanted, internally powered pulse generator (IPG), the word "battery" is used to refer to the entire device.

Biphasic (or multiphasic) stimulation - stimulation using waveforms in which an electrode contact(s) switches from cathode to anode (and/or vice versa) during one period or cycle of the stimulation waveform.

Bipolar (or multipolar) stimulation - stimulation using at least one cathode and at least one anode, typically of similar size, relatively near the target (see also Monopolar stimulation).

Burst stimulation - a series of stimulating pulses at a relatively high rate (frequency) repeated periodically at a lower rate; in some applications, the stimulation pattern is designed to mimic the pattern of naturally occurring action potentials observed in individual neurons; for example, five high frequency (500 per second) pulses followed by a pause, repeating 40 times per second.

Cathode - a stimulating contact programmed as a negative pole that attracts cations (positive ions). Compared with an anode, a cathode typically initiates action potential generation (depolarization) at a lower amplitude threshold; thus, cathodal effects predominate.

Center-surround physiology - a characteristic of the receptive fields and processing in many sensory systems including pain. This characteristic sharpens the signal-to-noise ratio and can be exploited for enhancing desired responses to neuromodulation.

Central point of stimulation - the effective center of a three-dimensional region of neural activation, typically predicted using computational methods and influenced by factors such as the location of anodes and cathodes, the impedance of intervening tissue(s), and waveform(s).

Channel - a path for current or signal to flow and pass. Multiple independent channels allow simultaneous delivery of pulses of different amplitudes to different contacts. (A generator that allows rapid sequential delivery of pulses to different contacts is not, strictly speaking, a multichannel device; instead, it is a "gated single channel" generator.)

Charge balance - equality of total positive and negative charge delivered in a given amount of time. Charge balance is necessary in neurostimulation applications because any net direct current (DC) can cause electrode and/or tissue damage. ${ }^{10}$ Balance can be achieved passively, for example, by a series capacitor that blocks DC, or actively by delivering compensatory charge (Fig. 2).

Charge density - the measure of electric charge per unit surface area of the electrode contact (or secondarily of tissue volume). In combination with charge per phase, charge density is a major determinant of electrochemical effects or toxicity.

Chronaxie - minimum time required for an electric current to double the strength of the rheobase to stimulate a muscle or neuron (see Rheobase).

Closed-loop stimulation - stimulation that adjusts automatically, typically in amplitude (including on-off), in response to certain inputs or feedback signals. Examples of these inputs include: (1) manifestations of disease, for example, detection of early or impending seizure activity, which can trigger therapeutic stimulation via brain electrodes, and (2) variables affecting the delivery of stimulation, such as postural changes in the distance from dorsal epidural electrodes to the spinal cord that are revealed by changes in evoked compound action potentials. Control loops may be combined or nested within other loops.

Compound action potential - compared with individual elements, multiple neurons or axons firing simultaneously will, in aggregate, produce a larger signal that can be recorded at a greater distance from their origin (for example, by unused electrode contacts).

Constant current (or current-controlled) stimulation - delivery of electrical charge at a variable voltage to maintain a uniform or desired rate of charge delivery even when changes occur in system impedance or resistance.

Constant voltage (or voltage-controlled) stimulation - delivery of charge at a variable rate (for example, coulombs per second) while maintaining a uniform or desired voltage even when changes occur in system impedance or resistance.

Contact - the electrically conductive portion of an electrode assembly at the interface with biological tissue, resembling and sometimes referred to as a "button." An individual contact can be programmed as an anode, a cathode, or neither (that is, off).

Contact combination - anode or cathode or on/off assignments of contacts in an array. Modern implanted electrodes have multiple contacts (Fig. 1).

Depolarization - a shift in electric charge distribution across the membrane of a cell, increasing the positive charge on the intracellular side and/or increasing the negative charge on the extracellular side.

Direct targeting - method of identifying the optimal location for electrode placement, particularly within the brain, using high-resolution imaging to visualize the target. 
Table 1. Continued

Dorsal column stimulation (DCS) - the original terminology for SCS, reflecting electrode placement directly over the dorsal columns as well as the relatively low threshold for recruitment of the large myelinated fibers therein. The term "DCS" went out of favor when it was recognized that SCS activates additional structures in the spinal cord.

Dose or dosage (also known as electrical dose) - the total or aggregate current or charge delivered by a stimulator, a product of frequency, pulse width, and amplitude. A major determinant of battery longevity or recharge interval, dose is also relevant to therapeutic and side-effects. Sometimes described as "neural dose," although glia and other structures are also affected.

Duty cycle - the ratio (commonly expressed as a percentage) of the sum of all pulse durations during a specified total period of delivery of a pulse train. For example, a train of $1 \mathrm{~ms}$ pulses at 100 per second has a duty cycle of $10 \%$. An alternative definition is the percentage of time that the device is turned on versus off, regardless of the pulse duration and frequency.

Efferent - nerve fiber(s) conducting or carrying signals from the central nervous system (brain/spinal cord) outward, for example, to peripheral effector tissues or organs.

Electrode - an assembly comprising electrical conductors (contacts, wire), insulating spacers, catheter(s), and backing material, in particular the part of the assembly that contains the contacts (see Contact). "Lead" (pronounced "leed," see Lead) is used synonymously but better describes the cable or wire leading between the electrode and the implanted pulse generator. In electrochemistry, "electrode" refers to a conductor that makes contact with a nonmetallic part of a circuit (that is, with tissue in neuromodulation applications).

Evoked compound action potential (ECAP) - an electrical signal that is measured or recorded as the result of summating multiple individual action potentials generated by a specific stimulus (see Action potential, Compound action potential, and Evoked potential).

Evoked potential (EP) - an electrical signal resulting from stimulation, such as a compound action potential or local field potential (LFP). EPS may be recorded from the brain, the spinal cord, and/or peripheral nerves, via surface (skin) electrodes or implanted electrodes (including unused contacts in an array), and they may be used to guide implantation and/or adjust stimulation.

Frequency - as commonly used in neurostimulation, which is delivered as a series of pulses, "frequency" (expressed in Hz) refers to pulse repetition rate (expressed in number of pulses per second). Strictly speaking, unless a sinusoidal waveform is used, each pulse contains a broad spectrum of frequencies, for example, the high frequency content of on and off transients with rectangular pulses.

Generator - a device that converts one form of energy into another, for example, chemical energy from a battery, whether external or internal, into electrical stimulation pulses delivered to implanted electrode(s).

Guarded cathode - an arrangement of contacts with two or more anodes bracketing or surrounding one or more cathodes. The boundary created by the anodes for the depolarizing effect of the cathode defines the area of nerve recruitment and thus of paresthesia. In general, patients treated with paresthesiabased SCS prefer a guarded cathode array.

High density stimulation - stimulation with relatively high frequency (typically as high as a given generator will allow, but not as high as $10 \mathrm{kHz}$ ) that increases the average current delivered and thus the "dose." The amplitude for high density stimulation is typically adjusted below (for example, to $75 \%$ of) that required to elicit paresthesia.

High frequency - pulse repetition rates at and above 1-1.5 kHz for SCS or above $100 \mathrm{~Hz}$ for deep brain stimulation (DBS). "High" is, of course, a relative term, and the meaning of "high frequency" has changed with context and over time.

Impedance - a measure of the opposition that a circuit presents to a current when a voltage is applied. Impedance extends the concept of electrical resistance from DC, which has only magnitude, to alternating current waveforms, in which the electrical resistance has both a magnitude and a phase at a given frequency.

Implanted pulse generator ("IPG" or "internally powered generator") - an implanted stimulation device containing a battery, to be distinguished from "radiofrequency (RF)" or "wireless" externally powered devices.

Indirect targeting - method of identifying the optimal location for electrode placement, particularly within the brain, using coordinates developed from standardized brain atlases relative to landmarks, such as the anterior-posterior commissural line.

Indifferent electrode (or contact) - the counterpart of an active electrode (or contact), providing a return path for stimulation current, typically remote from the target site (and often larger, thus reducing current density).

Interleave - to mix two or more stimulation pulse trains by alternating between them on a single channel or generator.

Laminectomy - removal of the bony roof of the spinal canal and associated ligamentous structures, providing access for placement of one or more electrodes, particularly insulated paddle or plate electrodes. The posterior midline spinous process is typically removed along with at least a portion of the lamina on either side to allow electrode placement in the midline.

Laminotomy - removal of part of at least one lamina to allow the introduction of an electrode.

Lateral stimulation - (defined physiologically) SCS affecting one side before the other or (defined anatomically) SCS delivered between the midline spinous processes and the medial border of the pedicles, that is, the lateral margin of the posterior epidural space.

Lead (pronounced "leed") - the insulated wire(s) or cable that connects (that is, leads from) an implanted generator or connector to the electrode contacts. In common usage, following the convention adopted for cardiac pacing and defibrillation devices, this term refers to the entire electrode assembly.

Local field potential (LFP) - an electrical potential recorded in the extracellular space that is the summed, synchronous electrical activity of individual neurons in the region.

Midline (spinal) - defined physiologically by stimulation, the right-left position at which a symmetric effect (for example, paresthesia) is achieved. Defined anatomically, the centerline of the spinous processes, which normally is midway between the pedicles and articular processes, as seen on a true anteriorposterior x-ray or fluoroscopic view.

Migration - displacement following implantation, particularly of an electrode, from its original or intended location. Commonly defined operationally as an event requiring surgical repositioning or replacement, with radiographic confirmation that the electrode has moved.

Monophasic (or unidirectional) stimulation - stimulation in which the current maintains the same direction or polarity throughout (although the amplitude can vary); any given contact will act only as a cathode or an anode (or be off).

Monopolar (or unipolar) stimulation - delivery of electric charge using one active contact near the stimulation target along with a remote indifferent contact (also called a "return" or "ground" contact), such as the metallic case of an implanted pulse generator. The active contact is typically a cathode and 
Table 1. Continued

the indifferent an anode, minimizing power requirements. "Monopolar" and "unipolar" are misnomers, however, in that two poles are required for stimulation.

Motor threshold - the minimum amplitude (at a given pulse width) at which stimulation elicits involuntary muscle contraction or twitch (which may be observed visually and/or palpated with each pulse at low pulse repetition rates).

Multichannel stimulator - a device that allows simultaneous delivery of pulses of different amplitudes to different contacts (to be distinguished from a multicontact system that allows rapid sequential delivery of pulses to different contacts).

Neuromodulation - modification of neurological function, including both neuronal and glial cell activity, through delivery of a stimulus, such as electrical stimulation, magnetic stimulation, or chemical agents, to specific neurological targets.

Nonlinear - lacking a first-order, proportional relationship between input and output or between independent and dependent variables.

Noninvasive electrical stimulation - stimulation applied with an external device only, that is, without any implanted component.

Open-loop stimulation - stimulation that can be turned on and off and adjusted by a patient or operator but is not adjusted automatically (see Closed-loop stimulation).

Orthodromic - the normal traveling direction of a signal in a nerve fiber.

Paresthesia - a tingling or buzzing sensation caused by stimulation.

Paresthesia-based SCS - SCS that causes a tingling or buzzing sensation. Even if subliminal stimulation, resulting in no perceived sensation at all, is to be used during treatment, paresthesia mapping can be useful to optimize electrode placement and contact selection.

Percutaneous or catheter electrode - an electrode that can be inserted through a needle, in a manner that is less invasive than implantation via laminectomy/laminotomy.

Period - the time it takes to complete one cycle of a stimulation waveform (the reciprocal of frequency or pulse repetition rate).

Peripheral nerve field stimulation - recruiting available, pertinent nerve fibers and terminals by placing percutaneous electrodes subcutaneously in an area of pain rather than targeting specific peripheral nerve(s).

Position (or postural) adaptive SCS - SCS that is automatically adjusted in amplitude to compensate for changes in the distance between the spinal cord and epidural electrodes by sensing changes in body position or in evoked compound action potentials. (Typically, amplitude requirements are lowest when the patient is supine).

Primary cell - a galvanic cell (battery or part of a battery) that cannot be recharged and must be replaced when drained.

Programmer - a person or device who/which adjusts the parameters of stimulation.

Pulse - a single stimulation waveform cycle or "phase" (Fig. 2).

Pulse width - the duration of a pulse, measured in microseconds (Fig. 2).

Radiofrequency (RF) - as commonly (and loosely) used in neuromodulation, this term is synonymous with "Wireless."

Receiver - an implanted, externally powered circuit that converts radiofrequency or microwave energy to electrical stimulation pulses and/or program settings.

Relative refractory period - the period shortly after the discharging of an action potential when partial repolarization has occurred and a greater/stronger than normal stimulus can elicit a second response.

Repolarization - the change in membrane potential that returns it to a more negative value just after the depolarization phase of an action potential has moved the membrane potential toward a positive value.

Resting potential - the voltage across the membrane of a neuron or other excitable cell relative to its surroundings when not stimulated or involved in the passage of an impulse.

Retrograde placement/access - placing electrodes or accessing the spine in a caudal direction (downward, toward the feet). This is opposite the typical placement of percutaneous electrodes cephalad (upward or "anterograde") in the dorsal epidural space.

Rheobase - the minimal stimulation amplitude of infinite duration that reaches the depolarization threshold, resulting in an action potential or the contraction of a muscle.

Sham stimulation - stimulation with an amplitude of zero (or effectively zero). In a scientific study, sham stimulation mimics delivery of active or therapeutic stimulation to provide a meaningful control for comparison, addressing potential nonspecific or placebo effects.

Stereotactic - a technique that uses coordinates defined by medical imaging to target a specific location or structure in the body, in particular the brain.

Strength duration - the relationship between the intensity of an electric stimulus and the length of time it is applied, that is, the pulse width, to achieve a given effect, for example, paresthesia.

Sub-perception (or subliminal) stimulation - stimulation that does not reach conscious perception. For example, SCS at sufficiently low amplitude and/or high frequency is not perceptible and is, therefore, subliminal and "paresthesia-free."

Surgical plate/paddle electrode - an array of contacts on an insulated curved or planar substrate implanted to face the spinal cord or nerve(s) or cortex. This arrangement reduces power consumption as well as extraneous stimulation that can cause side-effects, such as unwanted movement or pain. Because of their size and shape, paddle or plate electrodes must be inserted via an open surgical exposure.

Target (or sweet spot) - an anatomic or three-dimensional stereotactic location at which stimulation produces optimal effects, that is, maximal therapeutic effects and/or energy efficiency and/or minimal adverse or unwanted side-effects. Using SCS as an example, the target may be defined by maximal pain relief or by a surrogate, such as maximal paresthesia coverage of a painful area with minimal extraneous coverage. Using DBS as an example, it may be defined by maximal tremor suppression with minimal neuropsychiatric effects.

Threshold - the minimum amplitude at which an effect is observed. Perceptual threshold, for example, is commonly measured and expressed as the lowest voltage or current level at which a subject can feel any effect of stimulation, such as paresthesia (Fig. 3).

Tolerance - an increase in the dose requirement (for example, stimulation amplitude) for a given therapeutic effect, to be distinguished from therapy failure even at maximum available or feasible dose. Most often and most appropriately used in pharmacology.

Tonic (or monotonic) - a stimulation pulse train with parameters that do not vary from one pulse to the next - to be distinguished, for example, from burst stimulation, in which the pulse repetition rate changes periodically (Fig. 4). 
Table 1. Continued

Traditional (or conventional) SCS - tonic epidural stimulation of the dorsal spinal cord delivered in the tens and hundreds of $\mathrm{Hz}$ at an intensity that elicits paresthesia but remains below the motor or discomfort threshold.

Trial - temporary use of an implanted electrode to demonstrate satisfactory treatment results before proceeding with a permanent implant. For example, a DBS, PNS, or SCS electrode may be placed and used with a temporary external generator before incurring the expense and potential morbidity of a permanently implanted generator.

Virtual ground - a node in a circuit that is maintained at a constant reference potential without direct connection to the reference potential.

Waveform - a representation (usually graphical) of the variation of voltage or current amplitude over time (Figs. 2 and 3).

Wireless - a method of delivering energy or information (commands or telemetry) transcutaneously to and from an implant via an external transmitter and antenna (see also Radiofrequency).

and performance of neuromodulation technology. We included selected terms that have been given special attention by certain proprietary interests, but we did so only when the terms have a generally accepted meaning and have not become part of a brand name or trademark.

The format of the print edition, as it appears herein, reflects our desire to save space. Accordingly, we generally avoided the use of "see $x x$," with attendant duplication and greatly expanded word count, under the assumption that this document can and will be viewed and searched electronically.

\section{RESULTS}

Table 1 displays our glossary of neurostimulation terminology, and Table 2 lists neurostimulation acronyms, including some for terms in common use that are not included in our glossary. We archived additional terms and acronyms collected in parallel with the above for use in planned web editions but excluded them from this print edition because of size constraints.

\section{DISCUSSION}

The English language is complex and difficult to learn, but along with this burden comes the benefit of precision when it is used correctly. ${ }^{13,14}$ When language erodes or becomes corrupt, whether by design, inattention, or entropy, precision and utility are lost. Medical vocabulary draws from ancient languages, notably Latin and Greek, which can increase precision but also introduce confusion to the uninitiated, and efforts to standardize medical terminology have been met with difficulty. ${ }^{15}$ The field of neuromodulation requires a neuroscience and engineering vocabulary that, while specialized, must respond to the needs of an expanding base of writers, including those writing for the general public and those who represent commercial interests and payers with their own complex reimbursement and coding needs.

The expanding number of scientific disciplines and their settings (academia, industry, and so forth), in addition to ongoing developments in the field, has exerted pressure on neuromodulation terminology that has led to novel, creative, and at times inappropriate usage. For example, the recent introduction of new waveforms for SCS has led to use of the word "tonic" to distinguish conventional or traditional stimulation at frequencies in the tens or hundreds of $\mathrm{Hz}$ from higher frequencies. In this glossary, however, tonic is defined as a "stimulation pulse train with parameters that do not vary from one pulse to the next." As shown in Figure 4, therefore, "tonic" simply refers to a continuous train or sequence of stimulation pulses with invariant amplitude, duration, and frequency/repetition rate. This definition applies to all stimulation frequencies; thus, using "tonic" to distinguish between low and high frequency stimulation is incorrect. It is appropriate, however, to distinguish tonic from "burst" stimulation, in which the pulse repetition rate changes periodically and continually (Fig. 4).

Different neuromodulation procedures can use the same waveforms and/or devices (Fig. 1) and/or variations of the same surgical approach. For example, percutaneous trans-spinal placement of DRG stimulating electrodes begins with needle placement in the dorsal epidural space as is done for SCS. An abbreviated version of this language, however, can convey the incorrect impression that SCS is in fact being administered. ${ }^{16}$ Ambiguity sometimes facilitates regulatory, coding, and reimbursement objectives in our complex field ${ }^{17}$ viz, the billing codes for DRG and SCS are reportedly identical, $^{18}$ but we should encourage clarity in scientific communication.

Imprecise use of terms that have well understood meanings in other fields has arisen with adaptation of the terms to neuromodulation. A recent example is the introduction of the phrase "nonlinear burst stimulation" to describe a waveform that is distinguished primarily by passive charge balancing after each pulse and burst, as opposed to active or passive charge balancing after each pulse. ${ }^{19}$ The word "nonlinear" has a precise meaning in mathematics, statistics, and engineering and is applicable to a great many phenomena in neuromodulation but not useful in distinguishing between different forms of burst stimulation. $^{20}$

With ongoing developments in our field, the definitions of terms that have been well established are subject to change as well. For example, clinicians and researchers assumed for many years that pain relief by SCS would not occur below the threshold of perception of paresthesia; thus, therapeutic- as well as sideeffects were scaled to the range from this threshold up to the threshold of discomfort. ${ }^{11}$ In recent years, we learned that pain relief can occur below the threshold of perception, ${ }^{12}$ and this has become the expectation with some new waveforms. Likewise, side-effects, including discomfort, can and do occur above and below perceptual threshold. Figure 3 represents some of these relationships graphically. Ideally, we should not need to change any of our terminology to accommodate new findings; accordingly, we should take care to choose and use terms precisely and consistently.

As noted, we excluded trademarked terms from our glossary, but consistent with our goals, we were able to include terms that have received special attention from proprietary interests without being appropriated. A good example is "central point of stimulation," 


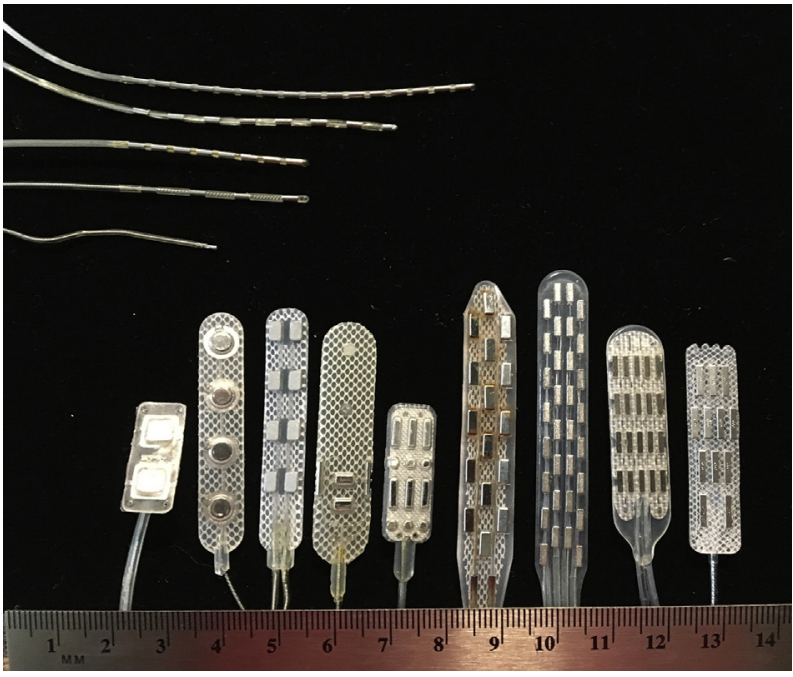

Figure 1. Representative multicontact electrodes approved for SCS may be inserted through a needle percutaneously (top) or implanted surgically (bottom); multiple percutaneous electrodes may be inserted in parallel to form twodimensional arrays resembling surgical electrodes. These and similar designs may be adapted (and sometimes are simply used off label) for PNS and other applications. [Color figure can be viewed at www.neuromodulationjournal.org]

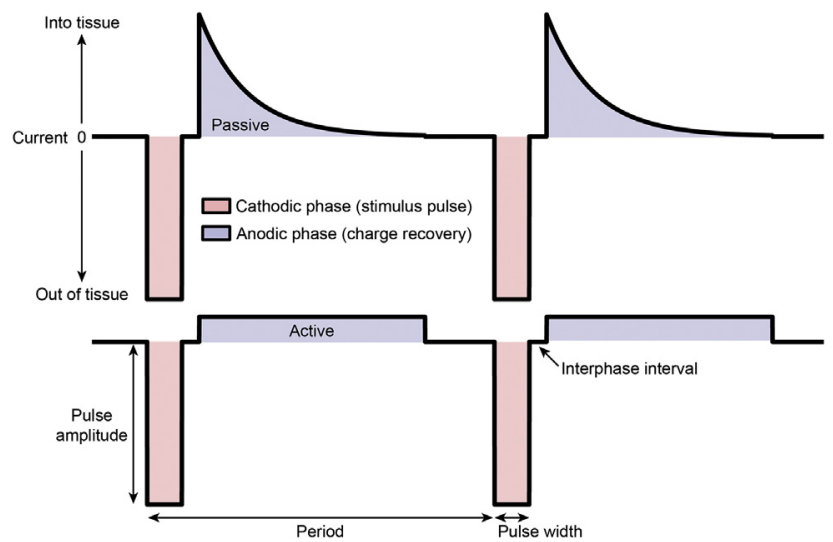

Figure 2. The amplitude or intensity of a typical stimulation pulse wave is measured in volts or milliamperes, its width or duration as well as period is measured in milliseconds or $\mu$ sec. In a charge balanced waveform, the areas between the baseline and the cathodic (negative, downward) and anodic (positive, upward) deflections and the baseline (charge per phase) must be equal. Charge balance can be achieved passively (by series capacitance in the circuit), or actively (by generating a second, compensatory pulse). [Color figure can be viewed at www.neuromodulationjournal.org]

which is currently used in SCS by one manufacturer but is also used in other applications (cochlear stimulation, noninvasive brain stimulation) by other entities. Likewise, the terms "density" (for example, charge density) and "dose" deserved inclusion, and we developed definitions from their self-evident meanings and published use.

The need for a glossary to address misguided usage and spelling has long been apparent. The word "paresthesia" provides an excellent example. Like "anesthesia," it has its roots in the Greek word for sensory perception. The addition of the prefix "an" comes from the Greek meaning "without," and the prefix "para" derives

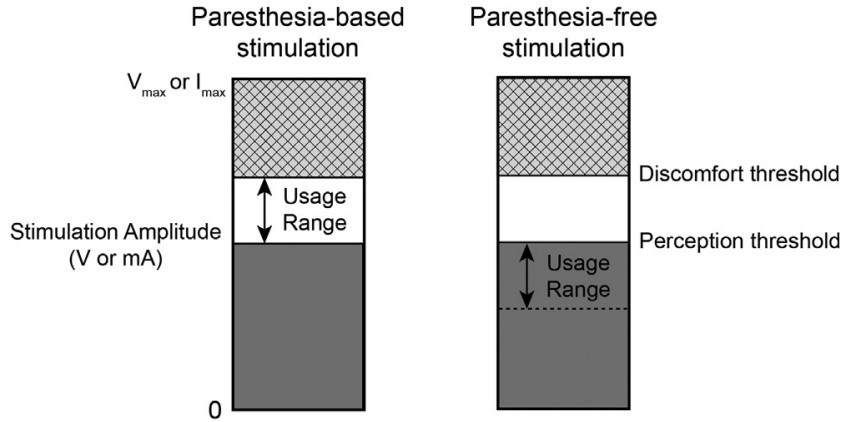

Figure 3. The therapeutic, clinically useful range of somatosensory stimulation (SCS and PNS) for pain was thought for many years to begin at the perceptual threshold, ${ }^{11}$ but we now understand that it can begin below that threshold, where the stimulation is "paresthesia free." ${ }^{\prime 2}$ As shown here, the useful upper limit is bounded by the discomfort threshold (which may be synonymous with the motor threshold), but in some applications, toxicity might determine the limit. The relationships of these thresholds vary for different waveforms and electrode positions; for example, discomfort can occur at the perceptual threshold

from the Greek for "beyond" or "beside" (as "paranormal" means "beyond normal"). Despite the lack of a similar plural for "anesthesia," some authors have ill-advisedly assumed that "paresthesia" has or needs a plural form such as "paresthesias" or even "paresthesiae." Like anesthesia, paresthesia may occur in more than one location; it may vary in its spatial extent, and it may come and go (for example, as stimulation cycles on and off), but "paresthesia" remains the only appropriate form and spelling.

IoN, the INS, and the NF will continue to collaborate on expanded online editions and updates.

\section{Limitations}

To date, we have limited the scope of this project to electrical stimulation of the nervous system with implanted devices. We have, thus, excluded terminology specific to magnetic (and electromagnetic) stimulation, optical stimulation, mechanical stimulation, and particularly pharmacological "stimulation" via implanted drug delivery systems.

The conditions that we treat with neurostimulation have become too numerous to include in this glossary. Other resources, such as neuromodulation textbooks and the INS website, ${ }^{21}$ provide information on neurostimulation indications. Likewise, we do not include the plethora of neuroscience and neurophysiology terms that are not specific to our field; these are also covered in textbooks and websites. ${ }^{5}$ We omitted some terms we deemed to be secondary or peripheral to save space in this print edition; we plan to include them in our online editions.

\section{CONCLUSIONS}

We have defined the need for a glossary of neuromodulation terms and have prepared the current print edition, expanded versions of which we plan to develop and maintain as an online resource. We believe this will facilitate communication among ourselves and with the public to the benefit of our growing field. We hope to mitigate inappropriate use of these terms in refereed papers and, thus, propagation in the neuromodulation literature. 


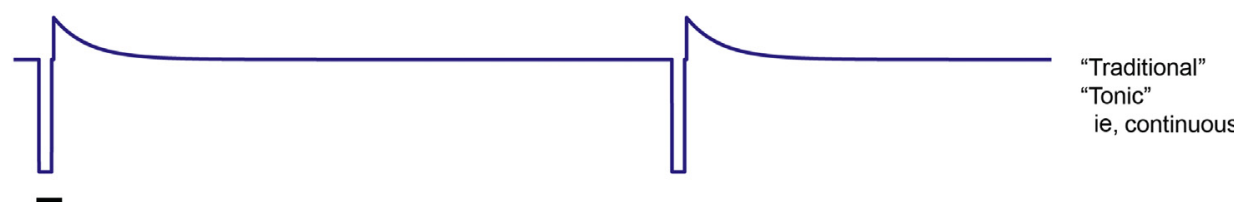

$1 \overline{\mathrm{ms}}$

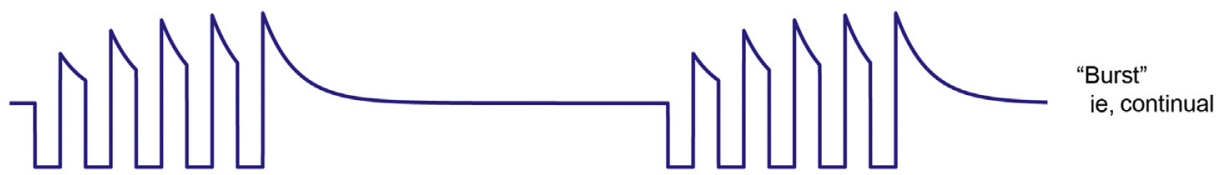

$1 \overline{\mathrm{ms}}$
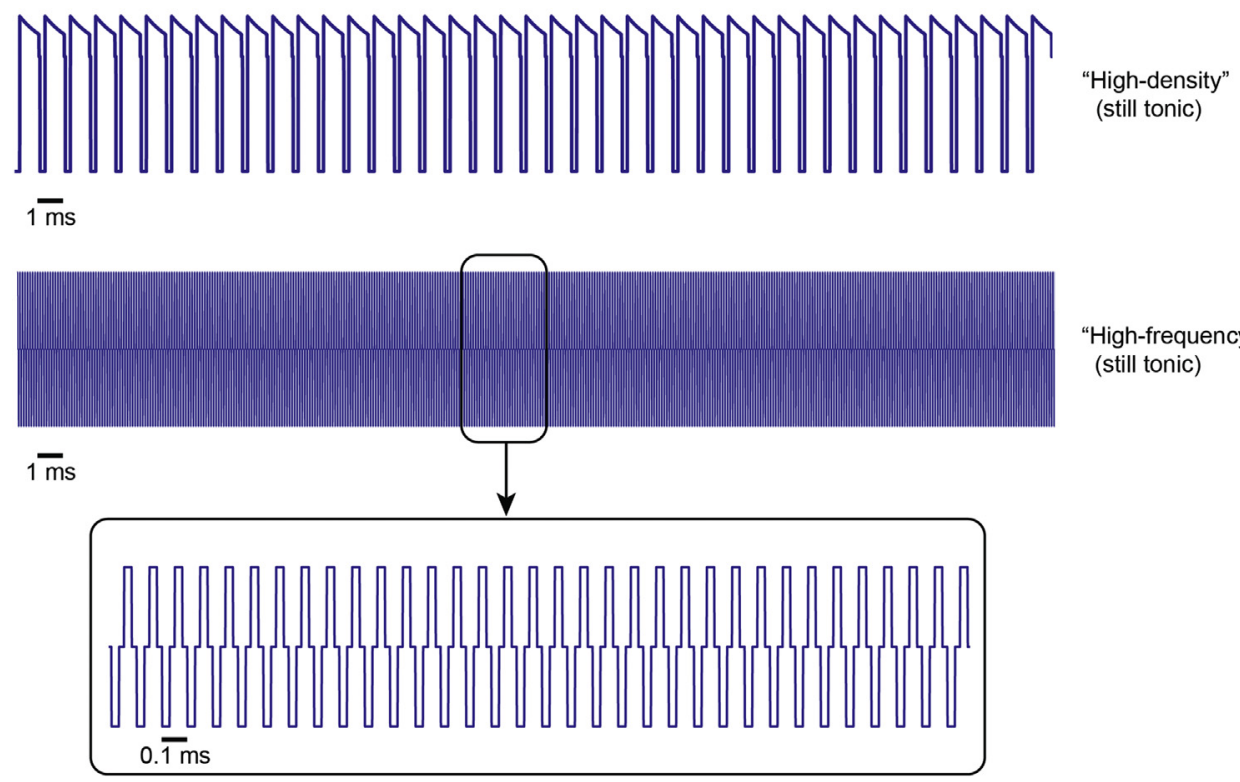

"High-frequency" (still tonic)

Figure 4. "Tonic" refers to a continuous train of stimulation pulses that are invariant from one pulse to the next; because this applies to all frequencies, the word should not be used to distinguish traditional, lower frequencies from novel high frequencies. It is appropriate, however, to distinguish tonic from "burst" stimulation, in which the pulse repetition rate changes periodically and continually. [Color figure can be viewed at www.neuromodulationjournal.org]

Table 2. Acronyms for Neurostimulation Terms.*

CNS - central nervous system

CSF - cerebrospinal fluid

DBS - deep brain stimulation

DC - direct current

DCS - dorsal column stimulation

DRG - dorsal root ganglion

ECAP - evoked compound action potential

$E P$ - evoked potential

$\mathrm{Hz}$ - Hertz

IPG - implanted pulse generator (or internally powered generator)

$\mathrm{kHz}-$ kilohertz

MCS - motor cortex stimulation

PNS - peripheral nerve stimulation

$\mathrm{RF}$ - radiofrequency

SCS - spinal cord stimulation

SNS - sacral nerve stimulation

VAS - visual analog scale

VNS - vagus nerve stimulation

*File modified from https://www.neuromodfound.org/wpsite/home/ glossary-acronyms/.

\section{Acknowledgements}

The authors thank the following for their contributions to this project: Jeffrey Ardell, PhD; Penney Cowan; Pierre-François D'Haese, PhD; Sam Eldabe, MD; Robert D. Foreman, PhD; Bert Joosten, PhD; Robert M. Levy, MD, PhD; Cameron Mclntyre, PhD; Kiran V. Patel, MD; P. Hunter Peckham, PhD; Marc Russo, MBBS, DA (UK), FANZCA, FFPMANZCA.

\section{Authorship Statements}

Richard B. North took the lead in conceptualization, methodology, data curation, project administration, writing the original draft, and validation and equal roles in visualization and final manuscript review. Jane Shipley had a supporting role in data curation, methodology, project administration, and writing the original draft and an equal role in editing the final manuscript. Scott F. Lempka, Yun Guan, Ellen L. Air, Lawrence R. Poree, Jeffrey Arle, Philippe Rigoard, and Simon Thomson had supporting roles in data curation, validation, and manuscript review. Scott F. Lempka also had an 
equal role in visualization. All authors approved the final version of the manuscript.

\section{How to Cite This Article:}

North R.B., Lempka S.F., Guan Y., Air E.L., Poree L.R., Shipley J., Arle J., Rigoard P., Thomson S. 2022. Glossary of Neurostimulation Terminology: A Collaborative Neuromodulation Foundation, Institute of Neuromodulation, and International Neuromodulation Society Project. Neuromodulation 2022; 25: 1050-1058.

\section{REFERENCES}

1. Hartley RVL. Transmission of information. Bell Syst Tech J. 1928;7:535-563.

2. North R, Shipley J, Prager J, et al. Practice parameters for the use of spinal cord stimulation in the treatment of chronic neuropathic pain. Pain Med. 2007;8(suppl 4):S200-S275.

3. The Neuromodulation Foundation. Glossary \& acronyms. https://www.neuromod found.org/treatment_of_chronic_neuropathic_pain/introduction/background/ glossary_acronyms/. Accessed August 10, 2021.

4. International Neuromodulation Society. Neuromodulation definitions. https:// www.neuromodulation.com/glossary-of-definitions. Accessed August 10, 2021.

5. Anon. Glossary. In: Purves D, George J, Augustine GJ, eds. Neuroscience. 5th ed Sinauer Associates; 2021.

6. Institute of Neuromodulation. https://neuromodulation.org/Default.aspx?TabID= 446. Accessed August 10, 2021

7. Fink A, Kosecoff J, Chassin M, Brook RH. Consensus methods: characteristics and guidelines for use. Am J Public Health. 1984;74:979-983.

8. Waggoner J, Carline JD, Durning SJ. Is there a consensus on consensus methodology? Descriptions and recommendations for future consensus research. Acad Med. 2016;91:663-668.

9. International Committee of Medical Journal Editors. Defining the role of authors and contributors. http://www.icmje.org/recommendations/browse/rolesand-responsibilities/defining-the-role-of-authors-and-contributors.html. Accessed August 10, 2021.

10. Merrill DR, Bikson B, Jefferys JG. Electrical stimulation of excitable tissue: design of efficacious and safe protocols. J Neurosci Methods. 2005;141:171-198.
11. Law JD, Miller LV. Importance and documentation of an epidural stimulating position. Appl Neurophysiol. 1982;45:461-464.

12. Yearwood TL. Neuropathic extremity pain and spinal cord stimulation. Pain Med. 2006;7:S97-S102.

13. Hoffman J. Corruption of the English language. Arizona Daily Star. https://tucson. com/opinion/local/jonathan-hoffman-corruption-of-the-english-language/ article 2481a1d3-3f25-5c78-8c3e-11b1d8874a46.html; 2018. Accessed August 10 2021.

14. Shipley JT. In Praise of English: The Growth \& Use of Language. The Crown Publishing Group: 1977.

15. Stevenson M, Guo Y. Disambiguation of ambiguous biomedical terms using examples generated from the UMLS metathesaurus. J Biomed Inform. 2010;43:762773.

16. Liem L, Russo M, Huygen FJ, et al. One-year outcomes of spinal cord stimulation of the dorsal root ganglion in the treatment of chronic neuropathic pain. Neuromodulation. 2015;18:41-49.

17. Raab GG, Parr DH. From medical invention to clinical practice: the reimbursement challenge facing new device procedures and technology-part 1: issues in medical device assessment. J Am Coll Radiol. 2006;3:694-702.

18. Mekhail N, Deer TR, Poree $L$, et al. Cost-effectiveness of dorsal root ganglion stimulation or spinal cord stimulation for complex regional pain syndrome. Neuromodulation. 2021;24:708-718.

19. Pope JE, Schu S, Sayed D, et al. Anatomic lead placement without paresthesia mapping provides effective and predictable therapy during the trial evaluation period: results from the prospective, multicenter, randomized, delivery study. Neuromodulation. 2020;23:109-117.

20. North RB. "Nonlinear" burst stimulation. Neuromodulation. 2020;23:260-261.

21. International Neuromodulation Society. Neuromodulation therapies-patient information. https://www.neuromodulation.com/therapies. Accessed August 10, 2021.

\section{COMMENT}

Although neuromodulation had been in practice for over 5 decades this is the first time a full glossary of terms has been assembled into one paper. This article will help reduce miscommunication between researchers and practitioners. It is a welcome addition to the literature.

Tracy Cameron, $\mathrm{PhD}$ Columbus, OH, USA 